\title{
Gridded Satellite (GridSat) GOES and CONUS data
}

\author{
Kenneth R. Knapp ${ }^{1}$ and Scott L. Wilkins ${ }^{2}$ \\ ${ }^{1}$ NOAA/NESDIS/National Centers for Environmental Information, Asheville, NC 28801, USA \\ ${ }^{2}$ Cooperative Institute for Climate and Satellites - North Carolina (CICS-NC), \\ North Carolina State University, Asheville, NC 28801, USA
}

Correspondence: Kenneth R. Knapp (ken.knapp@noaa.gov)

Received: 6 March 2018 - Discussion started: 27 April 2018

Revised: 17 July 2018 - Accepted: 18 July 2018 - Published: 6 August 2018

\begin{abstract}
The Geostationary Operational Environmental Satellite (GOES) series is operated by the US National Oceanographic and Atmospheric Administration (NOAA). While in operation since the mid-1970s, the current series (GOES 8-15) has been operational since 1994. This document describes the Gridded Satellite (GridSat) data, which provide GOES data in a modern format. Four steps describe the conversion of original GOES data to GridSat data: (1) temporal resampling to produce files with evenly spaced time steps, (2) spatial remapping to produce evenly spaced gridded data $\left(0.04^{\circ}\right.$ latitude), (3) calibrating the original data and storing brightness temperatures for infrared (IR) channels and reflectance for the visible channel, and (4) calculating spatial variability to provide extra information that can help identify clouds. The GridSat data are provided on two separate domains: GridSat-GOES provides hourly data for the Western Hemisphere (spanning the entire GOES domain) and GridSat-CONUS covers the contiguous US (CONUS) every $15 \mathrm{~min}$ (dataset reference: https://doi.org/10.7289/V5HM56GM).
\end{abstract}

\section{Introduction}

The National Oceanographic and Atmospheric Administration (NOAA) has used the Geostationary Operational Environmental Satellite (GOES) series since 1975 to monitor weather and other environmental conditions. While the series name has remained the same, the satellites providing the data have seen stepwise increases in capabilities. The original system was a spin-stabilized system. The system received a significant upgrade in 1994 with the launch of GOES 8 (the GOES-I to GOES-M series), providing a five-channel imager (Menzel and Purdom, 1994). The recent launch (in 2016) of GOES 16 is another substantial increase in capabilities (Schmit et al., 2017). The satellites have been maintained at two primary locations: GOES West near $135^{\circ} \mathrm{W}$, which observes the Eastern Pacific and western North America, and GOES East near $75^{\circ} \mathrm{W}$, providing coverage of North and South America and much of the Atlantic Ocean.

Gridded Satellite (GridSat) is produced as a means to facilitate access to GOES data. There are two domains provided: a 15 min domain provided over the contiguous US called
GridSat-CONUS and an hourly domain that spans the Western Hemisphere to cover the entire GOES domain, called GridSat-GOES. In the following, we use the term GridSatGOES to describe both these datasets, since their only difference is the temporal resolution and spatial coverage.

\subsection{Purpose for gridded GOES data}

Since the launch of the GOES-I to GOES-M series in 1994, worldwide computing capabilities have significantly improved and changed the landscape of how the data are transmitted, accessed, and used. When first launched, most GOES processing was conducted using the Man computer Interactive Data Access System (McIDAS, Lazzara et al., 1999) and data were transferred via satellite broadcast or high density tapes. Thus, data were used primarily for weather research and forecasts. About 25 years later, the data can be accessed with a variety of computers and software, primarily transferred over the internet and used for a myriad of purposes. Thus, the demand has significantly increased while the support of GOES formats has remained stagnant. Prior 
Table 1. Summary of GOES data access for AREA files.

\begin{tabular}{lll}
\hline & CLASS AREA files & Gridded GOES \\
\hline Format & AREA & netCDF-4 \\
Supported software languages & 1 (McIDAS) & $>30$ (supported at Unidata) \\
Navigation & Possible using thousands of lines of code & Stored directly in file \\
Calibration & IR: information available at NESDIS site & Stored directly in file \\
& Vis: available in scientific journals & \\
Temporal availability & Variable (1 to 30 min) & Regular (CONUS: 15 min, GOES: $1 \mathrm{~h})$ \\
Volume (1 month of data) & $\sim 563 \mathrm{~GB}$ & $\sim 45 \mathrm{~GB}$ \\
\hline
\end{tabular}

Table 2. Comparison of GridSat-GOES and GridSat-CONUS with similar currently available datasets.

\begin{tabular}{lllll}
\hline & Globally merged IR & GridSat-B1 CDR & GridSat-GOES & GridSat-CONUS \\
\hline Spatial coverage & Global $\left(60^{\circ} \mathrm{N}-60^{\circ} \mathrm{S}\right)$ & Global $\left(70^{\circ} \mathrm{N}-70^{\circ} \mathrm{S}\right)$ & Western Hemisphere & Contiguous US \\
Temporal coverage & 1998 -present & 1980 -present & 1994 -present & $1994-$ present \\
Spatial resolution & $\sim 4 \mathrm{~km}$ & $\sim 8 \mathrm{~km}$ & $\sim 4 \mathrm{~km}\left(0.04^{\circ}\right)$ & $\sim 4 \mathrm{~km}\left(0.04^{\circ}\right)$ \\
Temporal resolution & $30 \mathrm{~min}$ & 3 hourly & Hourly & 15 min \\
Spectral coverage & $1 \mathrm{IR})$ & 3 (IR, WV, VIS $)$ & $8^{2}(6$ channels and 2 others $)$ & $8^{2}(6$ channels and 2 others $)$ \\
Climate quality intercalibration & None & IR & None & None \\
Volume (1 month) & $45 \mathrm{~GB}$ & $12 \mathrm{~GB}$ & $45 \mathrm{~GB}$ & $12 \mathrm{~GB}$ \\
\hline
\end{tabular}

${ }^{1}$ Janowiak et al. (2001); ${ }^{2}$ see Sect. 4.1 for a description of the channels in the variable list.

to GridSat-GOES, the data were available from the NCEI archive as follows.

- Raw GVAR data - a very complex binary format intended for transmission of satellite data via satellite uplinks and downlinks. This can be read by a very limited set of software. The target audience are satellite experts with access to large computing resources.

- AREA format - a format developed for McIDAS to process satellite data. This is well documented, but still requires a thorough knowledge of satellite data to extend to other applications. The target audience are those with access to McIDAS software (e.g., academia).

- netCDF format - the netCDF file provided by the archive does not follow accepted standards in storing data and it provides no information on converting values to reflectance and brightness temperatures. There is also no obvious way to renavigate imagery. Therefore, while the target audience may be scientific users, these issues make the data provided by the archive less useful for that audience.

- TIFF and JPEG - these formats are solely for imaging. The data provided have map overlays. The target audience are the general public looking for satellite images for qualitative analysis, not quantitative studies.

What is lacking is a format that can be used by the general scientific community, such that it can easily be incorporated into other processes and used for analysis in a wide range of applications.
Table 1 contrasts the access to GOES data via McIDAS area files and the GridSat-GOES formats. The AREA file format is fully supported by one software application, the navigation (outside of McIDAS) requires more than 1000 lines of code, and the calibration information (outside of McIDAS) is not directly available in the AREA files, but requires accessing satellite-specific coefficients from another source. Lastly, the temporal resolution of the files can vary from 1 to $30 \mathrm{~min}$, with a complex scan schedule that varies depending on the scan mode selected for the day (e.g., when severe weather is expected, forecasters can request $1 \mathrm{~min}$ rapid scans of a region, at the expense of other regional scans). Conversely, the GridSat-GOES format provides calibrated and mapped data that has been temporally sampled to the nearest $15 \mathrm{~min}$ (for CONUS) and $1 \mathrm{~h}$ (for GOES). The format - netCDF-4 - is supported by Unidata, which provides information on how to use netCDF in multiple languages and applications.

\subsection{Relationship to other available datasets}

GridSat-GOES and GridSat-CONUS are most similar to two other datasets: the globally merged IR (infrared) dataset (Janowiak et al., 2001) and the GridSat-B1 Climate Data Record (CDR) (Knapp et al., 2011). Various details of the datasets are compared in Table 2. The global merged data has provided information for precipitation processing since the beginning of global meteorological geostationary satellite coverage in 1998, but only provides information from one channel: the infrared window. The GridSat CDR expands on this by providing intercalibrated IR brightness temperatures with a longer period of record (1980-present). The GridSat- 
GOES datasets focus on providing access to complete GOES data in a new, updated format. It provides access to all GOES Imager channels. Since it does not expand to other satellites, it is mostly limited to the Western Hemisphere.

\section{GOES data processing}

The effort to produce GridSat data required processing GOES area files retrieved from NOAA CLASS (Comprehensive Large Array-data Stewardship System). In so doing, we ordered and downloaded $294 \mathrm{~TB}$ of data in about 10 million files. This represents the entire archive of GOES-8 to GOES-15 data for 1994-2016. Processing these AREA files required several steps in order to produce the final GridSat products. The first step was to resolve the issue of duplicate files in the archive. This occurs when multiple files are available for one scan time. Next, specific scan times were selected. While the GOES scan schedule is a complex system of various scan schedules taking image scans (i.e., regions) at different times, the GridSat data provide grids at regular times. Image scans are then navigated, which involves determining the location of each pixel and, conversely, determining which image pixel is closest to a given Earth location. Once navigated, the scans are converted from digital counts with a 10 bit depth (hence they range from 0 to 1023) to geophysical units, either reflectance or brightness temperature, a process called calibration (note that visible data are not provided as reflectance factor, which would have a correction for the illumination geometry). Lastly, two other variables which provide information on the spatial uniformity of the images are calculated and stored in the resulting GridSat file. More details on these procedures are provided below.

\subsection{Resolving duplicate files}

Files were originally archived and stored on tapes. Each time a tape is read, there is the possibility that the file has slightly different contents. When this occurs, both files are retained and are differentiated by a trailing hyphen and number (e.g., “-1"). This produced duplicate files of the same image.

Duplicate files are prevalent in the GOES archive. They exist for 1994 through 2003 and can be up to $30 \%$ of the files for any given year. Because some files have more than one duplicate, it is not unusual to find files that are repeated 3 or 4 times. The most repeated file is "goes08.1995.060.174513" (from GOES 8 on 1 March 1995 at 17:45:13 UTC), which has six files for the single scan. The total number of extra files in the archive exceeds 64000 files; for context, that is more than the number of GOES files archived for 1997. In essence, there is the equivalent of an extra satellite year in the archive.

The reprocessing effort set up an objective algorithm to reconcile these duplicate files. Files were compared scan line by scan line. First, files that were identical were resolved simply by renaming or removing files. Where scan lines differed, the scan lines that were more highly correlated with neighboring scan lines were selected. This led to selecting one of the multiple files as the best; however, some cases resulted in a completely new file (when best scans were selected from different files). This process resolved all duplicated files.

\subsection{Temporal sampling}

The GOES Imager produces about 120 images per day; the exact number varies by scan schedule. Given the wide variety of scans and times, the GridSat-GOES and GridSatCONUS sectors store data at nominal 60 and 15 min intervals, respectively. That is, a sector that begins at 17:45 UTC will be included in the 17:45 UTC CONUS grid as well as the 18:00 UTC GOES grid. Thus, users do not need to be aware of the scan schedule, or understand where each is taken, in order to access and process GridSat-GOES data.

Furthermore, the hourly processing allows GridSat-GOES to merge images from various scans. The GOES scan schedule provides a full disk scan once every $3 \mathrm{~h}$. However, the various scans in the intermediate times, when merged, produce something that can approximate full disk coverage. Figure 1 provides a demonstration of this capability. Figure 1a shows an 18:00 UTC image, for which a full disk scan was available. However, in Fig. 1b, the sectors from multiple separate scans (Northern Hemisphere Extended, Southern Hemisphere, etc.) are merged on the same grid, thus providing a nearly full disk image.

The GridSat-GOES dataset provides a variable (called delta_time) that allows the calculation of the actual scan time of each pixel by storing the difference in time between the GridSat image time (17:45 and 18:00 UTC in the previous example) and the time of the actual instrument scan as derived from the scan line information in the AREA files. This allows for calculation of accurate solar illumination angles.

\subsection{Navigation}

Once a grid time is selected, the image pixels nearest each grid cell are selected through sampling. No averaging of the observations is performed. Sampling maintains the pixel bit depth, radiative characteristics, and spatial resolution. Data were navigated following the algorithms provided for GOES data by NESDIS (1998). Some navigation errors will be present in the remapped data from the original data; navigation accuracy for GOES is about $4 \mathrm{~km}$ during daylight and $6 \mathrm{~km}$ at night (Ellrod et al., 1998).

Since data are stored on a $0.04^{\circ}$ equal angle grid, simply displaying the data without transform, as in Fig. 1, provides an equirectangular map projection. 
(a)

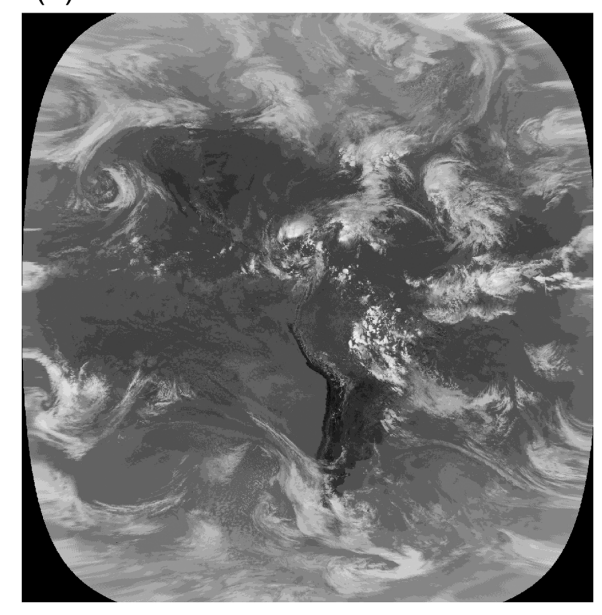

(b)

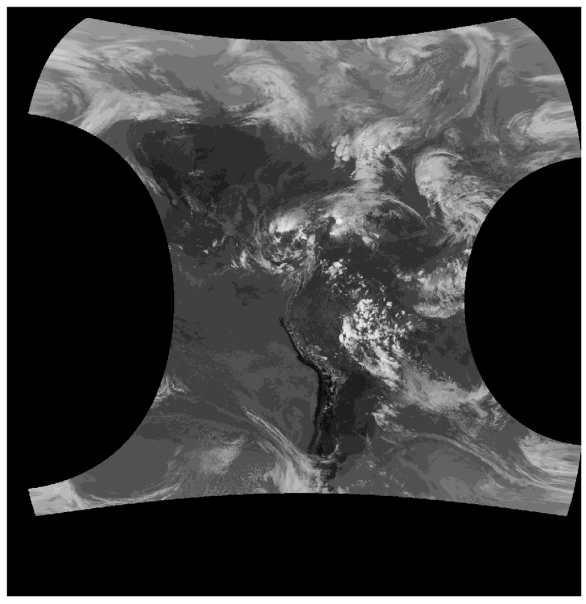

Figure 1. Sample of infrared window GridSat-GOES images for 18:00 and 19:00 UTC. (a) The 18:00 UTC image provided by a full disk scan that began at 17:45 UTC. (b) The 19:00 UTC image is a combination of three separate scans.

\subsection{Calibration}

Infrared channel data are calibrated following documentation from the NOAA STAR web page (Weinreb et al., 1997, 2017). Scan lines are calibrated separately and converted to a brightness temperature. The brightness temperatures are stored in two-byte integers using data packing (e.g., CF convention scale_factor and add_offsets). Brightness temperature noise is about $0.1 \mathrm{~K}$ at $300 \mathrm{~K}$ (Ellrod et al., 1998), which was improved to approximately $0.05 \mathrm{~K}$ at $300 \mathrm{~K}$ for imagers on GOES-13 to GOES-15 imagers (Zou et al., 2015).

There is no on-board visible calibration reference for any GOES satellite prior to GOES 16 , so some correction is necessary given the decay of the instrument gain (Knapp and Vonder Haar, 2000). Therefore, visible channel data are calibrated in GridSat-GOES following Inamdar and Knapp (2015) to provide a temporally stable set of visible observations.

\subsection{Visible and infrared window variability}

Lastly, two extra variables are provided: IR and visible channel variability. For each pixel that was mapped to a grid cell, the spatial variability is provided. The spatial variability is calculated as the standard deviation of the $3 \times 3$ pixels centered on the selected pixel. This is calculated at the original satellite resolution. Thus, for the visible channel, which is available at $1 \mathrm{~km}$ pixels, it provides a measure of sub-grid cell variability. For the IR, whose original resolution is near $4 \mathrm{~km}$, the calculation provides a measure of the grid cell variability. Both of these measures are related to cloud cover and can be used to determine the presence of clouds (Rossow and Garder, 1993).

\section{Future plans}

Many possible improvements exist. Future improvements will depend on user needs. Possible improvements include the following:

- Expansion to 1980-1994 - GridSat-GOES is currently limited to the GOES-I to GOES-M series of satellites (GOES 8 through GOES 15). The dataset can be expanded back in time to provide information from the previous series (GOES 1-7). While it would have fewer channels, it could maintain similar temporal and spatial resolutions.

- Expansion to GOES 16 and beyond - the newest series of GOES has the Advanced Baseline Imager (ABI), which increases the number of channels to 16 , increases the spatial resolution to $2 \mathrm{~km}$ for most channels, and the temporal resolution of full disk scans is $15 \mathrm{~min}$ (Schmit et al., 2005, 2017). Nonetheless, there will continue to be demand for this long-term GridSat series at a reduced volume. Expanding GridSat-GOES to GOES 16 could provide a solution for users needing large spans of time.

- Cloud information - including cloud information (probability, temperature, optical thickness, etc.) is possible and can be provided in future versions. While increasing the volume of the data, it would increase the usefulness to many users.

- Increase spatial resolution - visible data are available at $1 \mathrm{~km}$ spatial resolution and there has been some demand for that. However, it would significantly increase the volume (thus decreasing the usefulness of the data). One viable option would be to include the $1 \mathrm{~km}$ channel data only for the CONUS sector. 
- Merge GOES East and GOES West - data are presently provided separately for GOES East and GOES West. It is possible to merge the projections to one value, but doing so would impact data usefulness. In particular, the visible channel would be affected by including satellites in the same scene that have different viewing geometries.

- Climate quality calibration - presently, we are applying the operational brightness temperature corrections. There are higher quality calibrations that could be derived. While the visible channel calibration already includes values that provide longer-term stability, improvements could be made.

This is a short list of changes that could be made to further improve the utility of the data. User feedback will be used to prioritize these tasks and resources applied to those that are in highest demand.

\section{Data availability}

The data are available from the following links: GridSatCONUS, https://www.ncei.noaa.gov/data/gridsat-goes/ access/conus (Knapp, 2017); and GridSat-GOES, https: //www.ncei.noaa.gov/data/gridsat-goes/access/goes (Knapp, 2017). The web page https://www.ncdc.noaa.gov/gridsat/ (Knapp, 2018) provides additional GridSat-GOES information and allows user registration. Table 4 provides a list of files (for GridSat-GOES) provided by each satellite for each year. The most files possible in a year is 8760 files at 24 files per day (and 8784 in a leap year). The variation in numbers shows gaps in the record: large deviations from 8760 exist in the 1990s with more stable and relatively fewer missing files in the more recent years. This shows the progression of the GOES East position from GOES 8 to 12 to 13; a similar progression is apparent for GOES West. There are also numerous GridSat-GOES files for GOES 14; however, it was not listed in Table 3 since it never assumes a role as either GOES East or West for a significant time. The entire GridSat-GOES period of record for all satellites is $24 \mathrm{~TB}$, which is a factor of 10 smaller than the full-resolution archive (342 TB). The GridSat-CONUS period of record totals about $5 \mathrm{~TB}$ of data.

\subsection{Variables}

The following variables are provided in GridSat-GOES and GridSat-CONUS netCDF files. They are listed with a summary of how they could be used. A sample set of images is provided in Fig. 2 for GridSat-CONUS.

- Lat - the latitude coordinate variable (in ${ }^{\circ} \mathrm{N}$ ). This provides the ability to map the data.

- Lon - the longitude coordinate variable (in ${ }^{\circ} \mathrm{W}$ ), which also provides the ability to map the data.
Table 3. List of duplicate file numbers by year. The percentages are relative to the number of scans in the year.

\begin{tabular}{rr}
\hline Year & $\begin{array}{r}\text { Number of scans } \\
\text { with duplicate files }\end{array}$ \\
\hline 1994 & $448(3 \%)$ \\
1995 & $26137(41 \%)$ \\
1996 & $15830(22 \%)$ \\
1997 & $4743(7.6 \%)$ \\
1998 & $5(0.01 \%)$ \\
1999 & $37(0.04 \%)$ \\
2000 & $25(0.02 \%)$ \\
2001 & $41(0.04 \%)$ \\
2002 & $0(0 \%)$ \\
2003 & $153(0.15 \%)$ \\
\hline
\end{tabular}

- Time - nominal time for the GridSat data (in units of "days since 1 January 1970 00:00:00 UTC").

- Lat_bounds - from the CF convention, these provide the spatial bounds of the grid cells used.

- Lon_bounds - from the CF convention, these provide the spatial bounds of the grid cells used.

- Time_bounds - from the CF convention, these provide the temporal bounds of the temporal sampling.

- Satlat - the latitude of the subsatellite point (in ${ }^{\circ} \mathrm{N}$ ). This can be used in conjunction with satlon to calculate the viewing angles (e.g., satellite view zenith and azimuth angles).

- Satlon - the longitude of the subsatellite point (in $\left.{ }^{\circ} \mathrm{W}\right)$.

- Satrad - distance (in $\mathrm{km}$ ) of the satellite from the center of the Earth.

- Filename - filenames for the source archive AREA files included in the GridSat file, to provide lineage.

- Delta_time - provides the difference of the actual scan time (in minutes) from the optimal grid time, used for calculations of solar geometry.

- Ch1 - reflectance (or scaled radiance) for the $0.6 \mu \mathrm{m}$ band. The visible channel provides observations of clouds, Earth's surface, and aerosols.

- Ch2 - brightness temperature of the $3.9 \mu \mathrm{m}$ channel. This wavelength measures both reflected sunlight as well as the Earth's radiance and provides information on cloud microphysics and other surface information.

- Ch3 - brightness temperature of the $6.7 \mu \mathrm{m}$ channel, which is also called the water vapor channel. The channel provides information on the distribution and movement of water vapor in the upper atmosphere. The at- 


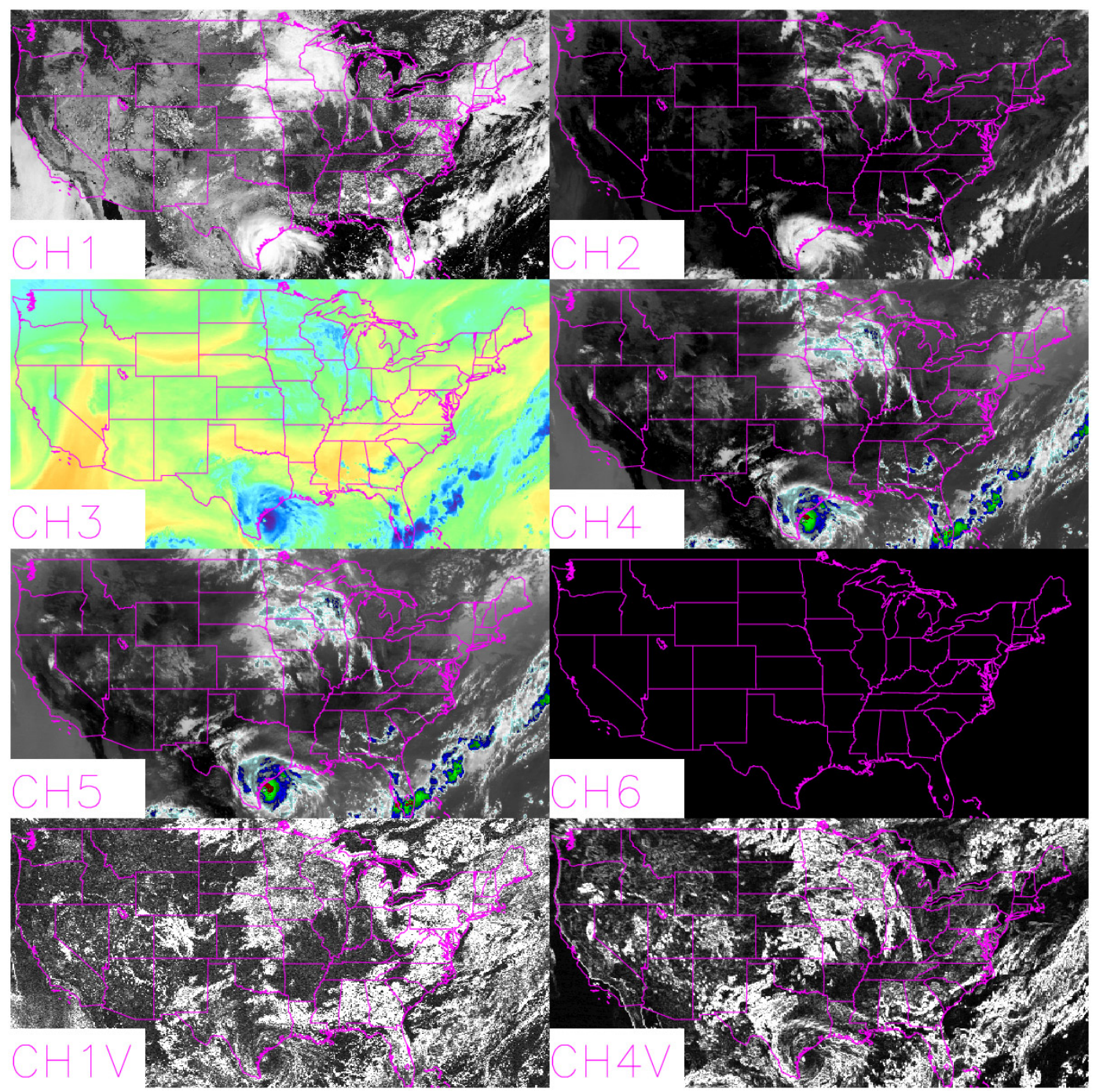

Figure 2. Sample images produced from GridSat-CONUS for 19 August 1999 at 17:45 UTC showing all eight channels. Since this is GOES 8, there is no channel 6 data; central wavelengths are provided in Sect. 4.1. Visible data are enhanced to show detail across the dynamic range.

mosphere at this wavelength is opaque and the channel rarely sees the surface.

- Ch4 - brightness temperature of the $11 \mu \mathrm{m}$ channel, generally called the IR window channel. It provides information on the radiant temperature on the surface or cloud top.

- Ch5 - brightness temperature of the $12 \mu \mathrm{m}$ channel. This channel provides information on the amount of atmospheric contamination in the $11 \mu \mathrm{m}$, which is why it is often called the split window. This channel is empty for GOES-12 to GOES-15 satellites because it is not included on those satellites.

- Ch6 - brightness temperature of the $13 \mu \mathrm{m}$ channel. This channel provides information on cloud cover and cloud height. This channel is empty for GOES-8 to GOES-11 satellites.

- Ch1v - spatial standard deviation of the channel 1 reflectance. This provides a measure of sub-grid cell variability because the calculation is made on the original satellite data, then sampled to the grid cells. Useful in identifying clouds (Rossow and Garder, 1993).

- Ch4v - spatial standard deviation of the channel 4 (infrared window) brightness temperatures, which is useful for identifying clouds.

As mentioned in Sect. 3, it could be possible to expand this variable list as deemed necessary to meet user needs. 
Table 4. Number of files per satellite per year for GridSat-GOES.

\begin{tabular}{|c|c|c|c|c|c|c|c|}
\hline \multirow[b]{2}{*}{ Year } & \multicolumn{3}{|c|}{ GOES East } & \multicolumn{4}{|c|}{ GOES West } \\
\hline & GOES 8 & GOES 12 & GOES 13 & GOES 9 & GOES 10 & GOES 11 & GOES 15 \\
\hline 1994 & 2316 & & & & & & \\
\hline 1995 & 8351 & & & 2324 & & & \\
\hline 1996 & 8143 & & & 6794 & & & \\
\hline 1997 & 7209 & & & 5903 & & & \\
\hline 1998 & 8418 & & & 4526 & 3816 & & \\
\hline 1999 & 8534 & & & & 8617 & & \\
\hline 2000 & 8581 & & & & 8692 & & \\
\hline 2001 & 8547 & & & & 8655 & & \\
\hline 2002 & 8557 & & & & 8655 & & \\
\hline 2003 & 2109 & 6511 & & & 8662 & & \\
\hline 2004 & & 8610 & & & 8655 & & \\
\hline 2005 & & 8585 & & & 8664 & & \\
\hline 2006 & & 8581 & & & 4075 & 4586 & \\
\hline 2007 & & 8277 & & & & 8647 & \\
\hline 2008 & & 8204 & & & & 8676 & \\
\hline 2009 & & 8287 & & & & 8353 & \\
\hline 2010 & & 2449 & 6268 & & & 8664 & \\
\hline 2011 & & & 8759 & & & 8040 & 609 \\
\hline 2012 & & & 8179 & & & & 8695 \\
\hline 2013 & & & 8280 & & & & 8757 \\
\hline 2014 & & & 8755 & & & & 8231 \\
\hline 2015 & & & 8759 & & & & 8753 \\
\hline 2016 & & & 8779 & & & & 8782 \\
\hline
\end{tabular}

(a)

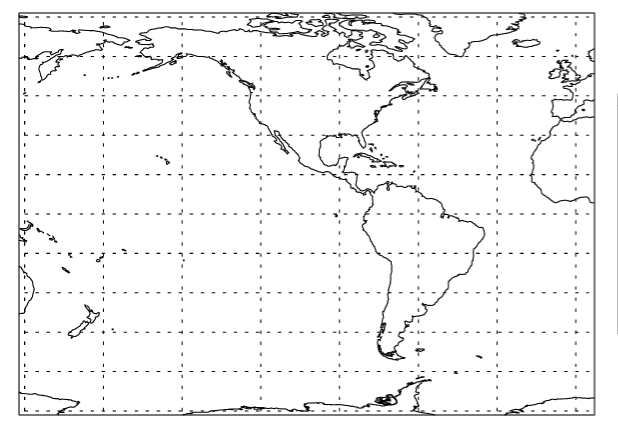

(b)

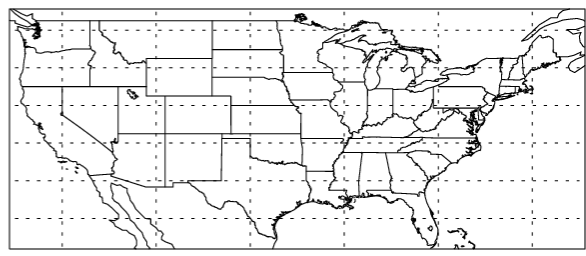

Figure 3. Spatial coverages of the $5375 \times 3750$ pixel domain of GridSat-GOES (a) and the $1500 \times 650$ pixel domain of GridSat-CONUS (b).

\subsection{Coverages}

The coverages of GridSat-GOES and GridSat-CONUS are shown in Fig. 3. GridSat-GOES spans the Western Hemisphere; in fact, it extends into the Eastern Hemisphere, spanning 150 to $5^{\circ} \mathrm{E}$ and spanning $75^{\circ} \mathrm{S}$ to $75^{\circ} \mathrm{N}$. The GridSatCONUS spans 125 to $65^{\circ} \mathrm{W}$ and 25 to $50^{\circ} \mathrm{N}$. Both sectors are provided at $0.04^{\circ}$, which is approximately $4 \mathrm{~km}$ near the Equator.

\subsection{Uncertainty}

Menzel and Purdom (1994) provide information on GOES calibration accuracy based on prelaunch design. Post-launch analysis has deemed IR calibration accurate to within 0.15 to $0.25 \mathrm{~K}$ (Ellrod et al., 1998; Wang et al., 2011). The visible calibration was provided by Inamdar and Knapp (2015), where the uncertainty is about $3 \%$ (in scaled reflectance units). 


\subsection{Caveats}

While the GridSat-GOES dataset is suitable for scientific and research use, some caveats should be considered prior to usage. First, users should become familiar with the limitations and quality of the source data: GOES Imager data (Ellrod et al., 1998; Goodman et al., 2018).

The IR calibration is not yet climate quality. We presently use the operational calibration without adjustment. There are, however, some issues that reduce the ability to use GridSatGOES for climate use. For instance, the midnight black body calibration correction was implemented by NOAA in 2003. Therefore, IR calibration is lower quality near the satellite local midnight prior to 1 April 2003. Also, the GSICS (Global Space-based Inter-Calibration System) community has worked on IR calibration adjustments (Goldberg et al., 2011), which are also not included in this initial version.

Users should also be aware of the limitations of the temporal and spatial sampling. The full-resolution visible data are sampled to the $0.04^{\circ}$ grid spacing. Similarly, full-resolution temporal scans (e.g., 1 min rapid scans) are used only when they provide an observation closest to the optimal grid time. Therefore, users requiring high spatial or temporal sampling will need to access GOES data using another method. Furthermore, no adjustments were made for parallax error, which are cloud location errors at high view zenith angles (Vicente et al., 2002).

Another limitation of the temporal sampling is the gaps that occur when data are not available. Three primary causes for the gaps are scan schedules, eclipse keep-out zones, and archive gaps. The scan schedule causes gaps mostly in the CONUS grid when the satellite is scanning the full disk (which takes longer than $15 \mathrm{~min}$ ). For example for GOES East, there is no 18:00 UTC GridSat-CONUS file since it is still doing a full disk scan that started at 17:45 UTC. These gaps generally occur at regular intervals and are usually limited to one missed CONUS grid. The keep-out zone refers to when the satellite is near the local midnight and has the potential to look directly at the Sun. Since this would permanently damage the satellite sensor and optics, a keep-out zone was implemented to reduce the possibility of damage. These are times near local midnight near the March and September equinoxes. The resulting gaps are daily and last a few hours. The GOES-13 to GOES-15 series includes an improved design that allows for shorter keep-out zones. Another limitation is the archive itself. There are some gaps in the archive where scans exist in other archives for one reason or another. These gaps can be longer than those caused by the scan schedule, often exceeding a day.

A complete list of known caveats and issues is maintained on the GridSat-GOES website.

\section{Conclusions}

The GridSat-GOES and GridSat-CONUS datasets provide GOES data to a wide audience in a standard format. Since the tasks of calibrating and navigating have been performed during production, data access is greatly simplified for the user. The temporal granularity structure is also much simpler, using fixed grid times rather than the complex and variable heritage scan times. The data are then made available in the widely supported netCDF-4 format. Future improvements and versions will be driven by user requirements.

Author contributions. KRK produced the GridSat data and wrote the manuscript. SLW ordered and managed the GOES data downloaded from NOAA CLASS.

Competing interests. The authors declare that they have no conflict of interest.

Acknowledgements. We would like to acknowledge Jessica Matthews and Anand Inamdar of CICS-NC for helping make this GOES reprocessing possible. This dataset would not have been produced without the computational support of CICS-NC. This work was supported by NOAA through the Cooperative Institute for Climate and Satellites - North Carolina under cooperative agreement NA14NES432003.

Edited by: David Carlson

Reviewed by: three anonymous referees

\section{References}

Ellrod, G. P., Achutuni, R. V., Daniels, J. M., Prins, E. M., and Nelson, J. P.: An Assessment of GOES-8 Imager Data Quality, B. Am. Meteorol. Soc., 79, 2509-2526, 1998.

Goldberg, M., Ohring, G., Butler, J., Cao, C., Datla, R., Doelling, D., Gärtner, V., Hewison, T., Iacovazzi, B., Kim, D., Kurino, T., Lafeuille, J., Minnis, P., Renaut, D., Schmetz, J., Tobin, D., Wang, L., Weng, F., Wu, X., Yu, F., Zhang, P., and Zhu, T.: The Global Space-Based Inter-Calibration System, B. Am. Meteorol. Soc., 92, 467-475, 2011.

Goodman, S. J., Schmit, T. J., Daniels, J., Denig, W., and Metcalf, K.: 1.05 - GOES: Past, Present, and Future, in: Comprehensive Remote Sensing, edited by: Liang, S., Elsevier, Oxford, 2018.

Inamdar, A. K. and Knapp, K. R.: Inter-comparison of Independent Calibration Techniques Applied to the Visible Channel of the ISCCP B1 Data, J. Atmos. Ocean. Tech., 32, 1225-1240, https://doi.org/10.1175/jtech-d-14-00040.1, 2015.

Janowiak, J. E., Joyce, R. J., and Yarosh, Y.: A real-time global half-hourly pixel-resolution infrared dataset and its applications, B. Am. Meteorol. Soc., 82, 205-217, 2001.

Knapp, K. R.: Gridded Satellite GOES (GridSat-GOES) East and West Full Disk and CONUS Coverage, Version 1, NOAA Na- 
tional Centers for Environmental Information, Asheville, NC, USA, https://doi.org/10.7289/V5HM56GM, 2017.

Knapp, K. R.: Gridded Satellite (GridSat) Data, https://www.ncdc. noaa.gov/gridsat/, last access: 1 August 2018.

Knapp, K. R. and Vonder Haar, T. H.: Calibration of the Eighth Geostationary Observational Environmental Satellite (GOES-8) Imager Visible Sensor, J. Atmos. Ocean. Tech., 17, 1639-1644, 2000.

Knapp, K. R., Ansari, S., Bain, C. L., Bourassa, M. A., Dickinson, M. J., Funk, C., Helms, C. N., Hennon, C. C., Holmes, C. D., Huffman, G. J., Kossin, J. P., Lee, H.-T., Loew, A., and Magnusdottir, G.: Globally gridded satellite (GridSat) observations for climate studies, B. Am. Meteorol. Soc., 92, 893-907, 2011.

Lazzara, M. A., Benson, J. M., Fox, R. J., Laitsch, D. J., Rueden, J. P., Santek, D. A., Wade, D. M., Whittaker, T. M., and Young, J. T.: The Man computer Interactive Data Access System: 25 Years of Interactive Processing, B. Am. Meteorol. Soc., 80, 271-284, 1999.

Menzel, W. P. and Purdom, J. F. W.: Introducing GOES-I: The first of a new generation of geostationary operational environmental satellites, B. Am. Meteorol. Soc., 75, 757-781, 1994.

NESDIS - National Environmental Satellite Data and Information Service: Earth Location User's Guide (ELUG) Revision 1, NOAA/NESDIS, Silver Spring, Maryland, USA, 1998.

Rossow, W. B. and Garder, L. C.: Cloud Detection Using Satellite Measurements of Infrared and Visible Radiances for ISCCP, J. Climate, 6, 2341-2369, 1993.

Schmit, T. J., Gunshor, M. M., Menzel, W. P., Gurka, J. J., Li, J., and Bachmeier, A. S.: Introducing the next-generation advanced baseline imager on GOES-R, B. Am. Meteorol. Soc., 86, 10791096, 2005.
Schmit, T. J., Griffith, P., Gunshor, M. M., Daniels, J. M., Goodman, S. J., and Lebair, W. J.: A Closer Look at the ABI on the GOES-R Series, B. Am. Meteorol. Soc., 98, 681-698, 2017.

Vicente, G. A., Davenport, J. C., and Scofield, R. A.: The role of orographic and parallax corrections on real time high resolution satellite rainfall rate distribution, Int. J. Remote Sens., 23, 221230, 2002.

Wang, L., Goldberg, M., Wu, X., Cao, C., Iacovazzi Jr., R. A., Yu, F., and Li, Y.: Consistency assessment of Atmospheric Infrared Sounder and Infrared Atmospheric Sounding Interferometer radiances: Double differences versus simultaneous nadir overpasses, J. Geophys. Res., 116, D11111, https://doi.org/10.1029/2010JD014988, 2011.

Weinreb, M. P., Jamieson, M., Fulton, N., Chen, Y., Johnson, J. X., Bremer, J. C., Smith, C., and Baucom, J. G.: Operational calibration of Geostationary Operational Environmental Satellite8 and -9 imagers and sounders, Appl. Optics, 36, 6895-6904, 1997.

Weinreb, M., Jamieson, M., Fulton, N., Chen, Y., Johnson, J. X., Bremer, J., Smith, C., and Baucom, J.: Operational Calibration of the Imagers and Sounders on the GOES-8 and -9 Satellites, http://www.ospo.noaa.gov/Operations/GOES/ calibration/calibration-paper/index.html, last access: 1 January 2017.

Zou, X., Qin, Z., and Zheng, Y.: Improved Tropical Storm Forecasts with GOES-13/15 Imager Radiance Assimilation and Asymmetric Vortex Initialization in HWRF, Mon. Weather Rev., 143, 2485-2505, 2015. 